\title{
Social Support and Socioeconomic Status Interact to Predict Epstein-Barr Virus Latency in Women Awaiting Diagnosis or Newly Diagnosed With Breast Cancer
}

\author{
Christopher P. Fagundes \\ The Ohio State University College of Medicine
}

Catherine M. Alfano

National Cancer Institute

\author{
Jeanette M. Bennett \\ The Ohio State University College of Dentistry
}

\begin{abstract}
Objective: Both higher socioeconomic status (SES) and supportive personal relationships confer health benefits, including better immune function. This study assessed the joint impact of SES and social support on the expression of a latent herpesvirus, Epstein-Barr virus (EBV), in a group of highly stressed women. Methods: Two-hundred and twenty four women either awaiting further evaluation following an abnormal mammogram or newly diagnosed with breast cancer completed questionnaires and provided blood samples to assess EBV viral capsid antigen (VCA) IgG antibody titers. Results: More highly educated women with more support from friends had lower EBV VCA antibody titers, reflecting a stronger cellular immune response to the latent virus; however, among less educated women, friend support was not associated with EBV antibody titers. As revealed in an ancillary analysis, more highly educated women with more friend support had lower systolic blood pressure (SBP); however, friend support was not associated with SBP among less educated women. Neither depression nor perceived stress mediated these associations. Neither cancer status nor cancer stage among those diagnosed with cancer was significantly related to these outcomes. Conclusion: Lower SES women may not reap the same immunological benefits from friend support when experiencing a stressful life event as their higher SES counterparts.
\end{abstract}

Keywords: socioeconomic status, cellular immunity, cancer survivorship, psychoneuroimmunology, psycho-oncology

Health disparities increase with each step down the socioeconomic status (SES) ladder (Adler \& Rehkopf, 2008). Adults with lower incomes, less education, and less prestigious jobs are at greater risk for chronic disease, disability, and premature mortality

This article was published Online First October 17, 2011.

Christopher P. Fagundes, Ronald Glaser, Stephen P. Povoski, Adele M. Lipari, Doreen M. Agnese, Lisa D. Yee, William E. Carson III, William B. Farrar, William B. Malarkey, Min Chen, and Janice K. Kiecolt-Glaser, College of Medicine, The Ohio State University; Jeanette M. Bennett, College of Dentistry, The Ohio State University; Catherine M. Alfano, National Cancer Institute.

Work on this project was supported by NIH grants CA131029, CA126857, DE014320, UL1RR025755, and CA016058, and a Postdoctoral Fellowship Grant from the American Cancer Society awarded to the first author PF-11-007-01-CPPB. We appreciate the helpful assistance of Arenda Nolan, Mary Lower, and Cathie Atkinson.

Correspondence concerning this article should be addressed to Christopher P. Fagundes, Institute for Behavioral Medicine Research, The Ohio State University, College of Medicine, 460 Medical Center Drive, Columbus, OH 43210. E-mail: christopher.fagundes@osumc.edu
(Stowe et al., 2010). Although income clearly limits health care access, the connection between lower SES and poor health still exists in countries with universal health care; thus, health care access is not the sole mechanism (Cohen, Doyle, \& Baum, 2006). Understanding why SES-related health disparities exist could lead to improvements in health.

Maladaptive alterations in immune function are central to SES health disparities. Research addressing pathogen burden has provided some of the strongest data in this regard, with the herpesviruses playing a pivotal role (Stowe et al., 2010). Once a person has been infected with one of the herpesviruses, they will carry the virus for the rest of their life (Glaser \& Kiecolt-Glaser, 1994). Herpesviruses create persistent latent infections, in which the virus remains "dormant" in latently infected cells. Under a variety of conditions, the virus may be triggered to reactivate in those cells and replicate, thereby producing new virus particles that kill cells. Persistent infections fuel chronic inflammatory responses, which in turn are linked to a range of age-related diseases (Steptoe et al., 2007).

SES researchers have focused on the Epstein-Barr virus (EBV), herpes simplex virus Type I (HSV-1), and cytomegalovirus 
(CMV) infections (Simanek, Dowd, \& Aiello, 2009; Steptoe et al., 2007; Stowe et al., 2010); these herpesviruses are ubiquitous in adults. More than $90 \%$ of adults are EBV seropositive (previously infected) (Glaser \& Kiecolt-Glaser, 1994), and more than $90 \%$ of individuals have antibody to HSV-1 by their 40s (Nahmias \& Roizman, 1973). Lower SES individuals are more likely to be infected with each of these herpesviruses earlier in life than higher SES individuals; they are also more likely to be seropositive for multiple pathogens and to show evidence of viral reactivation (Stowe et al., 2010).

Psychological stress and depression can drive herpesvirus reactivation or replication by impairing the ability of the cellular immune system to control viral latency (Glaser \& Kiecolt-Glaser, 1994). Both stress and depression are higher among those with lower incomes and less education (Adler \& Rehkopf, 2008). When the cellular immune system is compromised, EBV and other herpesviruses reactivate; the increased antiviral antibody production reflects the immune system's response to heightened herpesvirus replication (Glaser \& Kiecolt-Glaser, 1994).

Supportive interpersonal relationships can buffer the negative effects of stress on the cellular immune system and herpesvirus latency. For example, dementia spousal caregivers who reported lower levels of social support on entry into a longitudinal study showed greater negative changes in immune function a year later, including increases in EBV antibody titers (Kiecolt-Glaser, Dura, Speicher, Trask, \& Glaser, 1991). Lonelier medical students had higher EBV antibody titers than their fellow students who were not as lonely (Kiecolt-Glaser et al., 1984).

The experience of awaiting or receiving a breast cancer diagnosis is highly stressful, and supportive interpersonal relationships can buffer this distress (Hegel et al., 2006). Indeed, one study found that the elevated distress observed before a diagnostic breast biopsy remained high after the biopsy, regardless of the diagnostic outcome (Witek-Janusek, Gabram, \& Mathews, 2007). Supportive interpersonal relationships predict better adjustment among women diagnosed with breast cancer (Arora, Finney Rutten, Gustafson, Moser, \& Hawkins, 2007; Burgess et al., 2005; Epplein et al., 2010).

Lower SES individuals may not obtain the same stress reducing benefits from social support as higher SES individuals (Riley \& Eckenrode, 1986). Lower SES people receive lower quality support from others than those who are of higher SES (Belle, 1982, 1990; Krause \& Borawski-Clark, 1995). Higher SES individuals are more likely to seek support in times of high stress compared with those who are of lower SES (Krause, 1997). This difference is important because people benefit most from social support when they experience stress (Cohen \& Wills, 1985). Accordingly, SES may moderate the link between social support and health.

This study investigated how SES and social support were related to cellular immune function in a highly stressed sample of newly diagnosed breast cancer patients or those awaiting a potential breast cancer diagnosis. Specifically, we addressed the question of whether SES moderated the association between social support and EBV antibody titers. Psychological stress and depression can drive herpesvirus reactivation, and people benefit most from social support when stressed (Cohen, 2004; Cohen \& Wills, 1985). Given the heightened distress surrounding an abnormal mammogram or breast cancer diagnosis, we studied these relationships in both groups. Although we did not expect differential results between those awaiting a breast cancer diagnosis or newly diagnosed with breast cancer based on related studies (Witek-Janusek et al., 2007), we tested for these differences. As an ancillary analysis, we also assessed relationships between SES, social support, and blood pressure.

\section{Method}

\section{Participants}

Eligible women had an initial abnormal mammogram and were recruited prior to a diagnostic biopsy or immediately following a breast cancer diagnosis, allowing us to capture distressed women during diagnosis or soon thereafter. The women with benign diagnoses participated in the study prior to learning their cancer status; diagnoses were confirmed by medical records following participation. Screening exclusions included a prior history of breast or any other cancer except basal or squamous cell skin cancers. The institutional review board approved the project; all subjects gave written informed consent prior to participation.

Out of the 228 participants enrolled, three were EBV seronegative and one had not received a final diagnosis (i.e., benign or cancer); therefore, they were removed from analyses. Electronic medical records provided data on height and weight for all 224 women; they also provided blood pressure values within 6 weeks of the study visit for 143 women.

\section{Procedure}

One hundred and thirty-four women were recruited from outpatient surgical oncology clinics following a breast cancer diagnosis and participated prior to any treatment (i.e., surgery, radiation, or chemotherapy). Ninety women were recruited from surgical oncology and mammography clinics and participated prior to learning their cancer status. In this group of 90 women, 17 were subsequently diagnosed with cancer and 73 received a final benign diagnosis. Blood draws and questionnaire completion were conducted at the recruitment site or scheduled for a later time.

\section{Determination of EBV Viral Capsid Antigen (VCA) Immunoglobulin-G (IgG) Antibody Titers in Plasma}

EBV VCA IgG represents the antibody response to the combination of multiple viral proteins that make up the virus coat. We assessed antibody against EBV VCA IgG in plasma to assess control over viral latency. Plasma was stored at $-80^{\circ} \mathrm{C}$ until assayed with Euroimmun EBV ELISA plates (Boonton Township, NJ). This ELISA's antigen, a cell lysate of human B-cells infected with EBV strain P3HR-1 comprises various viral capsid proteins, including gp22, gp33, gp40, gp41, gp42, and gp116. EBV-VCA $\mathrm{IgG}$ antibody titers were assessed following instructions, with kit controls (one positive sample, one negative sample, and three calibrators) run in duplicate. After the initial 1:101 dilution, six serial twofold dilutions of each sample were assayed, and the last positive value was the IgG antibody titer. Calculated viral titers for each sample were plotted and samples were rerun if the endpoint did not fall within the linear range $( \pm 15 \%)$. 


\section{Measures}

Depression. The Center for Epidemiological Studies Depression Scale (CES-D) has been used extensively as a brief measure of depressive symptomatology (Basco, Krebaum, \& Rush, 1997; Radloff, 1977). Studies have shown acceptable test-retest reliability and excellent construct validity (Basco et al., 1997). As the CES-D has also distinguished depressed from nondepressed participants in community and clinical samples, discriminative validity appears acceptable as well (Basco et al., 1997). Population norms provide cutoffs for varying levels of depression (Basco et al., 1997), and it has been widely used in cancer studies (DemarkWahnefried et al., 2003). In this sample, Cronbach's alpha was .92.

Stress. The Perceived Stress Scale (Cohen, Kamarck, \& Mermelstein, 1983) was used to assess stress levels. It is designed to measure the degree to which individuals appraise situations in their life as stressful. In this sample, the 10-item short form was used and its coefficient alpha was .91.

Social support. The two subscales of the Perceived Social Support (PSS) Scale assess support from friends and family members. Higher scores reflect greater perceived social support (Procidano \& Heller, 1983). The alpha for the PSS-family subscale was .90 and the alpha for the PSS-friends subscale was .94. Separate assessments of friend and family support were desirable because there is evidence that the effects of friend and family support may differ across different demographic populations (Procidano \& Heller, 1983; Turner \& Marino, 1994).

Comorbidities. The Charlson index is the most widely used comorbidity index (Charlson, Szatrowski, Peterson, \& Gold, 1994). Originally developed for predicting mortality in breast cancer patients, it has now been widely used with both cancer and noncancer populations (Dobnig et al., 2008). Anyone who had more than 3 comorbidities was coded as a 3 , given that less than $5 \%$ of our sample exceeded this number.

Sleep. The Insomnia Severity Index (Morin, 1993) has been used reliably to collect data on insomnia severity (Bastien, Vallieres, \& Morin, 2001), including data from cancer patients (Savard, Savard, Simard, \& Ivers, 2005). Higher numbers indicate greater insomnia severity. In this sample, Cronbach's alpha was .89 .

Demographic and clinical variables. Participants answered questions about their age, race, highest level of education, marital status, smoking status, weekly average alcohol consumption, and current medication use. Following participants' authorization, electronic medical records were reviewed to obtain blood pressure, height, and weight data; body mass index (BMI; $\mathrm{kg} / \mathrm{m}^{2}$ ) was calculated from height and weight data. Breast cancer stage data were obtained through the Cancer Registry or electronic medical records.

Educational level was used to assess SES because $40 \%$ of the women in our sample did not work outside the home. In addition, education is less vulnerable to fluctuations than current income and job status (Gorman \& Sivaganesan, 2007; Marmot et al., 1998; Winkleby, Jatulis, Frank, \& Fortmann, 1992). Women chose from the following options: junior high only, some high school, high school graduate, some college, college graduate, or graduate/ professional school.

\section{Analytic Method}

Education was modeled as a continuous variable based on prior work showing that the association between SES and health is monotonic (i.e., the association between SES and health shows a gradient increase) (Adler et al., 1994; Gallo, Bogart, Vranceanu, \& Matthews, 2005). A $\log _{10}$ transformation normalized the distribution of EBV antibody titers. Zero-order correlations assessed relationships between education, social support, EBV antibody titers, systolic blood pressure (SBP), diastolic blood pressure (DBP), age, BMI, comorbidities, depressive symptoms, and alcohol consumption. Chi-square tests were conducted to assess group differences among dichotomous variables. Analyses of variance (ANOVAs) tested for group differences on all continuous variables. Among cancer patients, ANOVAs assessed breast cancer stage group differences on EBV antibody titers, SBP, DBP, depression, family support, friend support, and education.

Using separate ordinary least squares multiple regression, we addressed the question of whether SES interacted with social support to predict each study outcome. All independent variables were grand mean centered. We examined residuals to confirm that they distributed normally. To test for moderation, family support, friend support, education, and the interactions between family support and education, and friend support and education were entered as predictors.

For each analysis, we ran both unadjusted and adjusted regression models. When predicting EBV antibody titers in the adjusted models, we controlled for age, BMI, sleep, alcohol consumption, and comorbidities as linear, and cancer status $(1=$ breast cancer, $0=$ benign control $)$ and smoking status $(1=$ current smoker, $0=$ nonsmoker) as indicator variables. In the adjusted models predicting blood pressure, we also controlled for blood pressure medication ( $1=$ user, $0=$ nonuser). SBP and DBP were analyzed in separate models. In the model predicting depressive symptoms, we adjusted for age, cancer status, and comorbidities. To test if relationships between SES and social support could be explained by depression or stress, we added depressive symptoms and perceived stress (independently) as a final step of the models predicting EBV antibody titers and blood pressure.

\section{Results}

As shown in Table 1, women with breast cancer had more comorbidities than women in the benign group, consistent with the fact that the former all had a cancer diagnosis. In addition, women with breast cancer reported more family and friend support than women in the benign group. Smoking status, race, marriage status, all $\chi^{2} \mathrm{~s}(1, N=224)<.75, p s>.39$, and blood pressure medication, $\chi^{2}(1, N=139)=.22, p=.64$, were not significantly related to the cancer status group. The analyses comparing those who knew they had cancer with the group awaiting a cancer diagnosis produced identical results to the analyses comparing those who had breast cancer with benigns; the group that knew they had a cancer diagnosis mirrored the breast cancer group, while the group awaiting a cancer diagnosis mirrored the benign group.

Among those diagnosed with cancer, breast cancer stage was not related to the primary variables of interest: EBV antibody titers, depression, family support, friend support, or education, all $F \mathrm{~s}(4$, $138)<1.67, p s>.16$, nor systolic or diastolic blood pressure, all 
Table 1

Sample Population Characteristics

\begin{tabular}{|c|c|c|c|c|c|c|c|c|c|c|}
\hline \multirow[b]{2}{*}{ Variable } & \multicolumn{3}{|c|}{ Total $(N=224)$} & \multicolumn{3}{|c|}{ Cancer $(N=151)$} & \multicolumn{3}{|c|}{ Benign $(N=73)$} & \multirow[b]{2}{*}{$F^{a}$} \\
\hline & $\%$ & $M$ & $S D$ & $\%$ & $M$ & $S D$ & $\%$ & $M$ & $S D$ & \\
\hline $\operatorname{EBV}\left(\log _{10}\right)$ & & 3.09 & 0.39 & & 3.09 & 0.41 & & 3.09 & 0.37 & .011 \\
\hline Age (years) & & 55.21 & 11.63 & & 55.21 & 11.83 & & 55.19 & 11.28 & .000 \\
\hline BMI $\left(\mathrm{kg} / \mathrm{m}^{2}\right)$ & & 28.96 & 6.92 & & 28.90 & 6.95 & & 29.08 & 6.90 & .032 \\
\hline $\mathrm{SBP}(\mathrm{mmHg})$ & & 132.98 & 20.31 & & 134.35 & 20.86 & & 128.59 & 18.03 & 2.100 \\
\hline DBP $(\mathrm{mmHg})$ & & 80.01 & 9.77 & & 80.21 & 10.51 & & 79.35 & 6.98 & .199 \\
\hline Race (Caucasian) & 83 & & & 82 & & & 85 & & & \\
\hline Married & 66 & & & 66 & & & 64 & & & \\
\hline Sleep & & 8.49 & 5.70 & & 8.50 & 5.87 & & 8.47 & 5.37 & .001 \\
\hline Weekly alcohol consumption & & 2.08 & 3.96 & & 1.87 & 3.82 & & 2.52 & 4.21 & .362 \\
\hline Smoker & 14 & & & 15 & & & 11 & & & \\
\hline Comorbidities & & 1.10 & 0.88 & & 1.48 & 0.70 & & 0.32 & 0.66 & $139.86^{*}$ \\
\hline Blood Pressure medication (yes) & 35 & & & 36 & & & 32 & & & \\
\hline \multicolumn{11}{|l|}{ Education } \\
\hline Junior high & 1 & & & 1 & & & 0 & & & .442 \\
\hline Some high school & 4 & & & 3 & & & 4 & & & \\
\hline High school & 23 & & & 24 & & & 20 & & & \\
\hline Some college & 19 & & & 22 & & & 14 & & & \\
\hline College graduate & 29 & & & 23 & & & 41 & & & \\
\hline Graduate/professional & 24 & & & 27 & & & 21 & & & \\
\hline \multicolumn{11}{|l|}{ Breast cancer stage } \\
\hline 0 & & & & 18 & & & & & & \\
\hline I & & & & 34 & & & & & & \\
\hline II & & & & 34 & & & & & & \\
\hline III & & & & 10 & & & & & & \\
\hline IV & & & & 4 & & & & & & \\
\hline \multicolumn{11}{|l|}{ Perceived support } \\
\hline Family & & 16.50 & 4.98 & & 16.95 & 4.68 & & 15.56 & 5.46 & $3.862^{\mathrm{t}}$ \\
\hline Friend & & 15.64 & 4.39 & & 16.13 & 4.06 & & 14.63 & 4.88 & $5.901 *$ \\
\hline Depression & & 14.24 & 10.82 & & 15.06 & 10.99 & & 12.55 & 10.32 & 2.673 \\
\hline Stress & & 14.78 & 7.52 & & 14.96 & 7.86 & & 14.41 & 6.78 & .262 \\
\hline
\end{tabular}

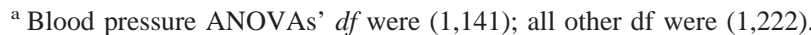

${ }^{\mathrm{t}} p<.10$. $^{*} p<.05$. $^{* * * *} p<.001$.

$F_{\mathrm{s}}(4,102)<1.43, p \mathrm{~s}>.23$. The average CES-D score was over $14 ; 44.6 \%$ of the sample scored 16 or higher, the clinical depression cut-off (Radloff, 1977; Roberts \& Vernon, 1983). Our sample was clearly highly distressed.

Table 2 provides correlations for all linear variables. Neither SBP nor DBP was associated with EBV antibody titers. Neither depression nor perceived stress was associated with EBV antibody titers, SBP, or DBP. Education was not directly related to either friend or family support.

Table 3 summarizes the analyses that assessed whether education and social support interacted to predict EBV antibody titers. Although the interaction between family support and education

Table 2

Correlations Among All Study Variables

\begin{tabular}{|c|c|c|c|c|c|c|c|c|c|c|c|c|}
\hline Variable & 1 & 2 & 3 & 4 & 5 & 6 & 7 & 8 & 9 & 10 & 11 & 12 \\
\hline \multicolumn{13}{|l|}{ 1. Education } \\
\hline 2. Family Support & -.02 & & & & & & & & & & & \\
\hline 3. Friend Support & .06 & $.54^{* * * *}$ & & & & & & & & & & \\
\hline 4. EBV $\left(\log _{10}\right)$ & -.06 & -.07 & $-.11^{\mathrm{t}}$ & & & & & & & & & \\
\hline 5. Systolic BP & $-.16^{\mathrm{t}}$ & -.02 & -.10 & .12 & & & & & & & & \\
\hline 6. Diastolic BP & -.08 & .05 & .10 & .07 & $.63^{* * * * *}$ & & & & & & & \\
\hline 7. Age & -.09 & -.04 & -.08 & .08 & $.28^{* *}$ & -.07 & & & & & & \\
\hline 8. BMI & $-.18^{* *}$ & -.10 & -.06 & .07 & $.32^{* * * * *}$ & $.23^{\text {*** }}$ & .06 & & & & & \\
\hline 9. Comorbidities & -.08 & $.11^{\mathrm{t}}$ & $.12^{\mathrm{t}}$ & .06 & $.20^{*}$ & .09 & $.24^{* * * *}$ & .03 & & & & \\
\hline 10. Depression & .02 & $-.28 * * *$ & $-.28^{* * * * *}$ & .10 & .01 & .03 & $-.28^{* * * *}$ & .06 & .04 & & & \\
\hline 11. Stress & .00 & $-.27^{* * * *}$ & $-.24^{* * * *}$ & .02 & -.05 & .05 & $-.22^{* * *}$ & .01 & .01 & $.78^{* * * *}$ & & \\
\hline 12. Weekly alcohol consumption & $.22^{* *}$ & -.02 & .01 & -.07 & .01 & .12 & $-.11^{\mathrm{t}}$ & $-.25^{* * * *}$ & -.08 & .01 & -.04 & \\
\hline 13. Sleep & -.10 & $-.25^{\text {**** }}$ & $-.18^{* * *}$ & .09 & -.02 & .04 & $-.13^{\mathrm{t}}$ & -.04 & .02 & $.61^{\text {**** }}$ & $.52^{* * * *}$ & -.01 \\
\hline
\end{tabular}

${ }^{\mathrm{t}} p<.10 . \quad{ }^{*} p<.05 . \quad{ }^{* * *} p<.01 .{ }^{* * * *} p<.001$. 
Table 3

Summary of Unadjusted and Adjusted Regression Analyses Predicting EBV Antibody Titers

\begin{tabular}{|c|c|c|c|c|}
\hline Model & Variable & $B$ & $S E$ & $95 \%$ confidence interval \\
\hline \multirow[t]{7}{*}{ Unadjusted } & Family support & -.002 & .006 & {$[-.014, .011]$} \\
\hline & Friend support & -.008 & .007 & {$[-.022, .007]$} \\
\hline & Education & -.013 & .022 & {$[-.056, .030]$} \\
\hline & Family Support $\times$ Education & .001 & .006 & {$[-.011, .012]$} \\
\hline & Friend Support $\times$ Education & $-.013^{*}$ & .006 & {$[-.026,-.001]$} \\
\hline & $R^{2}$ & .039 & & \\
\hline & $F(5,218)$ & 1.766 & & \\
\hline \multirow[t]{14}{*}{ Adjusted } & Age (years) & .003 & .003 & {$[-.002, .008]$} \\
\hline & BMI $\left(\mathrm{kg} / \mathrm{m}^{2}\right)$ & .003 & .004 & {$[-.005, .011]$} \\
\hline & Smoking status & .076 & .082 & {$[-.085, .238]$} \\
\hline & Weekly alcohol consumption & -.024 & .032 & {$[-.088, .039]$} \\
\hline & Sleep & .007 & .005 & {$[-.003, .016]$} \\
\hline & Cancer Status & -.008 & .074 & {$[-.154, .138]$} \\
\hline & Comorbidities & .028 & .041 & {$[-.053, .109]$} \\
\hline & Family support & .001 & .007 & {$[-.012, .014]$} \\
\hline & Friend support & -.008 & .007 & {$[-.023, .006]$} \\
\hline & Education & .004 & .023 & {$[-.041, .050]$} \\
\hline & Family Support $\times$ Education & .001 & .006 & {$[-.011, .013]$} \\
\hline & Friend Support $\times$ Education & $-.014^{*}$ & .006 & {$[-.027,-.002]$} \\
\hline & $R^{2}$ & .065 & & \\
\hline & $F(12,211)$ & 1.226 & & \\
\hline
\end{tabular}

$* p<.05$.

was not significant, friend support interacted with education to predict EBV antibody titers in both the unadjusted and adjusted models. Specifically, more friend support was significantly associated with lower EBV antibody titers among more highly educated women (see Figure 1); however, this was not the case among
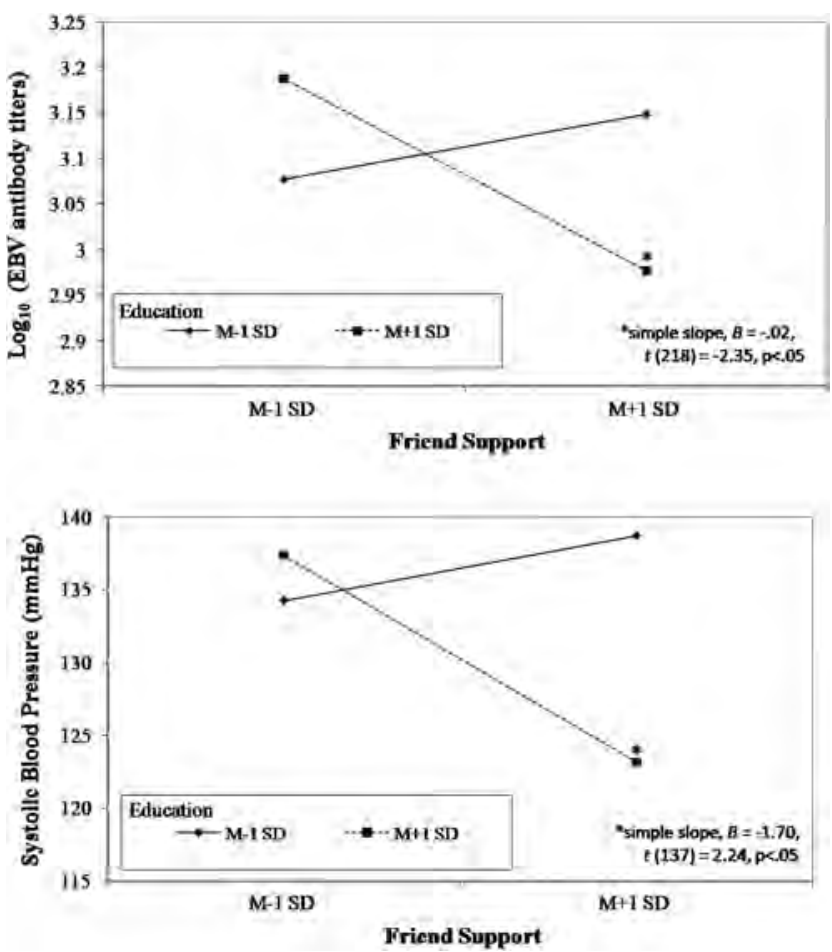

Figure 1. The moderating role of education in the relationship between friend support and EBV antibody titers and SBP. less educated women. Neither depressive symptoms nor perceived stress mediated this interaction.

As can be seen in Table 4, for both the unadjusted and adjusted models, there was no significant interaction between family support and education predicting SBP. Similar to the results for EBV antibody titers, friend support and education interacted to predict SBP. Specifically, more friend support was associated with lower SBP when level of education was high (see Figure 1); however, this was not the case when level of education was low. Neither depressive symptoms nor perceived stress mediated this interaction. For DBP, neither family support nor friend support interacted with SES.

Table 5 summarizes the analysis with depressive symptoms as a dependent variable. Those who reported less family and friend support had more depressive symptoms. Education was not associated with depressive symptoms and it did not interact with family or friend support in predicting depressive symptoms.

Post hoc analyses revealed no higher order interactions between cancer status and the reported associations. Likewise, there were no higher order interactions between those who knew they had a positive cancer diagnosis and those who were awaiting a cancer diagnosis. Given that neither SBP nor DBP was associated with EBV antibody titers (previously mentioned), blood pressure could not mediate the interaction between education and social support predicting EBV antibody titer levels. For all analyses, family and friend support were also modeled separately because of their high correlation; the pattern of results did not change.

\section{Discussion}

The connection between SES and physical health is a notable public health concern, and a better understanding of the factors that contribute to these disparities is important (Blane, 1995). This study addressed the joint impact of social support and SES (in- 
Table 4

Summary of Unadjusted and Adjusted Regression Analyses Predicting Systolic and Diastolic Blood Pressure

\begin{tabular}{|c|c|c|c|c|c|c|c|}
\hline \multirow[b]{2}{*}{ Model } & \multirow[b]{2}{*}{ Variable } & \multicolumn{3}{|c|}{ SBP } & \multicolumn{3}{|c|}{ DBP } \\
\hline & & $B$ & $S E$ & $95 \%$ confidence interval & $B$ & $S E$ & $95 \%$ confidence interval \\
\hline \multirow[t]{7}{*}{ Unadjusted } & Family support & .212 & .449 & {$[-.676,1.100]$} & -.028 & .220 & {$[-.463, .406]$} \\
\hline & Friend support & -.478 & .506 & {$[-1.479, .522]$} & .289 & .247 & {$[-.201, .778]$} \\
\hline & Education & -2.266 & 1.449 & {$[-5.130, .589]$} & -.613 & .709 & {$[-2.014, .788]$} \\
\hline & Family Support $\times$ Education & .359 & .419 & {$[-.471,1.188]$} & .160 & .205 & {$[-.246, .566]$} \\
\hline & Friend Support $\times$ Education & $-.944^{*}$ & .450 & {$[-1.834,-.055]$} & -.324 & .220 & {$[-.759, .112]$} \\
\hline & $R^{2}$ & .064 & & & .033 & & \\
\hline & $F(5,137)$ & 1.878 & & & .930 & & \\
\hline \multirow[t]{14}{*}{ Adjusted } & Age (years) & $.399^{*}$ & .166 & {$[.070, .727]$} & -.076 & .084 & {$[-.241, .090]$} \\
\hline & BMI $\left(\mathrm{kg} / \mathrm{m}^{2}\right)$ & $.875^{* * *}$ & .247 & {$[.387,1.364]$} & $.398^{* *}$ & .124 & {$[.151, .644]$} \\
\hline & Smoking status & 4.356 & 4.969 & {$[-5.477,14.189]$} & 2.616 & 2.506 & {$[-2.344,7.576]$} \\
\hline & Weekly alcohol consumption & 2.640 & 2.003 & {$[-1.323,6.603]$} & $1.919^{\mathrm{t}}$ & 1.010 & {$[-.080,3.918]$} \\
\hline & Cancer status & 4.052 & 4.475 & {$[-4.804,12.907]$} & .547 & 2.257 & {$[-3.919,5.014]$} \\
\hline & Cardiovascular medications & 4.877 & 3.914 & {$[-2.869,12.623]$} & 2.106 & 1.974 & {$[-1.802,6.013]$} \\
\hline & Comorbidities & 1.309 & 2.548 & {$[-3.735,6.352]$} & .482 & 1.285 & {$[-2.062,3.026]$} \\
\hline & Family support & .095 & .419 & {$[-.733, .924]$} & .022 & .211 & {$[-.396, .440]$} \\
\hline & Friend support & -.046 & .487 & {$[-1.010, .917]$} & .300 & .246 & {$[-.186, .786]$} \\
\hline & Education & -.877 & 1.417 & {$[-3.681,1.928]$} & .120 & .715 & {$[-1.295,1.534]$} \\
\hline & Family Support $\times$ Education & .313 & .387 & {$[-.453,1.078]$} & .113 & .195 & {$[-.273, .499]$} \\
\hline & Friend Support $\times$ Education & $-.983^{*}$ & .422 & {$[-1.817,-.148]$} & $-.363^{\mathrm{t}}$ & .213 & {$[-.784, .058]$} \\
\hline & $R^{2}$ & .272 & & & .166 & & \\
\hline & $F(12,126)$ & $3.929^{* * * *}$ & & & $2.086^{*}$ & & \\
\hline
\end{tabular}

${ }^{\mathrm{t}} p<.10 .{ }^{*} p<.05 .{ }^{* * *} p<.01 .{ }^{* * * *} p<.001$.

dexed by education) in women who were dealing with the significant stress of a potential or an actual breast cancer diagnosis. More highly educated women who had more support from friends had lower EBV antibody titers, reflecting better cellular immune function; however, for less educated women, friend support was not associated with EBV antibody titers.

The current findings add to our understanding of the potential underlying mechanisms behind SES disparities seen in cancer survivors and the general population. In general, more supportive interpersonal relationships have been associated with better cellu- lar immunity (Kiecolt-Glaser et al., 1991; Kiecolt-Glaser et al., 1987). In terms of friend support, our results suggest this may not be the case for those who are of lower SES. SES and social support may not have been directly related to EBV antibody titers because of the strength of this interaction. Indeed, due to the high levels of distress found in our sample, it is not surprising that depressive symptoms and perceived stress did not predict EBV antibody titers.

Elevated antibody titers to a latent herpesvirus reflect poorer cellular immune system control over virus latency (Henle \& Henle,

Table 5

Summary of Regression Analysis Predicting Depression

\begin{tabular}{clccc}
\hline \multicolumn{1}{c}{ Variable } & $B$ & $S E$ & $95 \%$ confidence interval \\
\hline Unadjusted & Family support & $-.380^{*}$ & .169 & {$[-.713,-.047]$} \\
& Friend support & $-.437^{* * *}$ & .189 & {$[-.810,-.065]$} \\
& Education & .296 & .570 & {$[-.827,1.420]$} \\
& Family Support $\times$ Education & -.053 & .158 & {$[-.365, .259]$} \\
& Friend Support $\times$ Education & -.169 & .168 & {$[-.499, .162]$} \\
& $R^{2}$ & .109 & & \\
Adjusted & $5.360^{* * * *}$ & & \\
& $F$ & $-.300^{* * * *}$ & .059 & {$[-.416,-.184]$} \\
& Age (years) & 2.135 & 2.027 & {$[-1.860,6.130]$} \\
& Cancer status & 1.802 & 1.372 & {$[-.904,4.507]$} \\
& Comorbidities & $-.399^{*}$ & .158 & {$[-.712,-.087]$} \\
& Family support & $-.558^{* *}$ & .178 & {$[-.910,-.207]$} \\
& Friend support & .169 & .535 & {$[-.726,1.519]$} \\
& Education & -.054 & .149 & {$[-.348, .240]$} \\
& Family Support $\times$ Education & -.175 & .158 & {$[-.486, .137]$} \\
& Friend Support $\times$ Education & .232 & & \\
\hline$R^{2}$ & $8.103^{* * * *}$ & & \\
& $F$ & &
\end{tabular}

${ }^{*} p<.05 . \quad{ }^{* * *} p<.01 . \quad{ }^{* * * *} p<.001$. 
1981), and thus provide one broad marker of cellular immune system function. The elevated antibody titers that signal poorer control over viral latency are most often asymptomatic, but not benign. Recent research has highlighted links between herpesvirus reactivation and inflammation. For example, a viral protein synthesized during EBV replication can enhance production of proinflammatory cytokines IL-6, TNF- $\alpha$, and IL-1 $\beta$ (Glaser et al., 2006). Elevated CMV antibody titers have been associated with increased IL-6 and TNF- $\alpha$ production (Roberts, Haan, Dowd, \& Aiello, 2010). Thus, inflammatory responses to viral replication may serve to increase proinflammatory cytokine production.

Among more highly educated women, more friend support, but not family support, was associated with better cellular immunity. Most social support scales do not differentiate between friend and family support (Gottlieb \& Bergen, 2009). During a cancer experience, support from friends may be particularly beneficial as family members are likely dealing with their own shock and fears (Cassileth et al., 1985; Compas et al., 1994; Edwards \& Clarke, 2004). Furthermore, family members are expected to be a source of support, while an outpouring of support from friends may come as a welcome surprise. One study showed that breast cancer survivors reported more satisfaction with the support they received from friends than family members (Neuling \& Winefield, 1988). As described by others, higher SES friends are able to provide higher quality support than their lower SES counterparts (Belle, 1982, 1990; Krause \& Borawski-Clark, 1995). In addition, friends may not be able to protect lower SES women against other chronic stressors that disproportionately affect them and may also affect immune function (e.g., economic strain, multiple social demands, other sick family members, etc.).

Education can provide an entrée into peer groups that provide higher quality support, while simultaneously promoting positive health behaviors and self-efficacy (Winkleby et al., 1992). Accordingly, our SES measure might be tapping into the quality of support these women's friends are able to provide, while other SES measures may not. For example, subjective social status may be more indicative of one's perceived position within a social network than the quality of support received from that social network (Matthews \& Gallo, 2011).

The current findings are in accord with work suggesting that SES is an important moderator of the relationship between social support and health outcomes such that higher SES individuals are more likely to benefit from social support than those with fewer resources (Gorman \& Sivaganesan, 2007). Other SES studies have produced different results. For example, one study found that those with lower incomes actually benefited more from support than those with higher incomes (as indexed by high-density lipoproteins and natural killer cell activity) (Vitaliano et al., 2001). However, half of that sample consisted of spousal caregivers whose caregiving duties likely made them more vulnerable to changes in both their income and social network. Another study found that those with lower education, but not higher education, had lower IL-6 if they also reported more positive relations with others (Morozink, Friedman, Coe, \& Ryff, 2010). Yet the positive relationship measures used in this investigation tapped into people's ability to trust others rather than their perceptions of support.

EBV antibody titers did not differ between our breast cancer and benign control participants. In accord with our data, a large prospective case-control study also showed that EBV VCA IgG antibody titers did not differ between breast cancer patients and controls prior to cancer diagnosis (Cox et al., 2010). However, chemotherapy can depress cellular immune function, a process which promotes herpesvirus reactivation (Kuo et al., 2008). In one study with cancer patients who were assessed before chemotherapy and then followed through treatment, both CMV IgG antibody titers and CMV viral load in leukocytes rose steeply during chemotherapy (Kuo et al., 2008). Two proinflammatory cytokines, TNF- $\alpha$ and IFN- $\gamma$, also increased substantially following reactivation, but not before (Kuo et al., 2008). Thus, premorbid differences in herpesvirus latency and inflammation could be exacerbated by cancer treatment.

The interaction between friend support and education predicting SBP mirrored the interaction predicting EBV antibody titers. SBP is an important predictor of cardiovascular disease (Kannel, 1999). Both elevated blood pressure and higher levels of EBV antibody titers are associated with higher hypothalamic-pituitary-adrenal (HPA)-axis and sympathetic activity (Cacioppo et al., 2002; Connell et al., 1987; Grassi, Cattaneo, Seravalle, Lanfranchi, \& Mancia, 1998; Lin \& Dean, 1984). Accordingly, alterations in autonomic and HPA-axis functioning may be the shared physiological mechanism underlying these parallel findings. Our blood pressure results were preliminary in nature and should be interpreted with caution given that they were unavailable for many of the participants and taken from medical charts at varying times within the assessment.

We focused exclusively on women who were newly diagnosed with breast cancer or awaiting a breast cancer diagnosis; thus, we do not know if our findings generalize to men, one limitation of our study. Furthermore, our sample was predominately White, another limitation of our study that should be addressed in future work with a more diverse sample. Although our sample was more educated than the general population, we were nonetheless able to detect theoretically consistent results. It is possible that our findings would be even stronger if we had been able to study more women who were less educated. Education was the best SES index for our sample because a large proportion of the women were older and did not work outside the home. However, future research with different samples would ideally examine how other SES indices such as job status also influence these outcomes.

In conclusion, our data suggest that less educated women may not reap the same immunological benefits from friend support as their more educated counterparts. This difference may be important because cancer treatment can diminish cellular immunity (Kuo et al., 2008). Future research should address how cellular immune differences observed following diagnosis contribute to subsequent health disparities during cancer treatment and recovery. These findings add to our growing understanding of how SES disparities influence health.

\section{References}

Adler, N., Boyce, T., Chesney, M., Cohen, S., Folkman, S., Kahn, R., \& Leonard, S. (1994). Socioeconomic status and health. American Psychologist, 49, 15-24. doi:10.1037/0003-066X.49.1.15

Adler, N., \& Rehkopf, D. (2008). Us disparities in health: Descriptions, causes, and mechanisms. Public Health, 29, 235. doi:10.1146/ annurev.publhealth.29.020907.090852

Arora, N., Finney Rutten, L., Gustafson, D., Moser, R., \& Hawkins, R. (2007). Perceived helpfulness and impact of social support provided by 
family, friends, and health care providers to women newly diagnosed with breast cancer. Psycho Oncology, 16, 474-486. doi:10.1002/ pon. 1084

Basco, M. R., Krebaum, S. R., \& Rush, A. J. (1997). Outcome measures of depression. In H. H. Strupp, L. M. Horowitz, \& M. J. Lambert (Eds.), Measuring patient changes in mood, anxiety, and personality disorders (pp. 207-245). Washington D. C.: American Psychological Association. doi:10.1037/10232-007

Bastien, C. H., Vallieres, A., \& Morin, C. M. (2001). Validation of the insomnia severity index as an outcome measure for insomnia research. Sleep Medicine Reviews, 2, 297-307. doi:10.1016/S13899457(00)00065-4

Belle, D. (1982). The stress of caring: Women as providers of social support. In L. Golderberger \& S. Breznitz (Eds.), Handbook of Stress: Theoretical and Clinical Aspects (pp. 496-505). New York: Free Press.

Belle, D. (1990). Poverty and women's mental health. American Psychologist, 45, 385-389. doi:10.1037/0003-066X.45.3.385

Blane, D. (1995). Social determinants of health-socioeconomic status, social class, and ethnicity. American Journal of Public Health, 85, 903. doi:10.2105/AJPH.85.7.903

Burgess, C., Cornelius, V., Love, S., Graham, J., Richards, M., \& Ramirez, A. (2005). Depression and anxiety in women with early breast cancer: Five year observational cohort study. British Medical Journal, 330, 702-702. doi:10.1136/bmj.38343.670868.D3

Cacioppo, J., Kiecolt-Glaser, J., Malarkey, W., Laskowski, B., Rozlog, L., Poehlmann, K., ... Glaser, R. (2002). Autonomic and glucocorticoid associations with the steady-state expression of latent Epstein-Barr virus. Hormones and Behavior, 42, 32-41. doi:10.1006/hbeh.2002.1801

Cassileth, B., Lusk, E., Strouse, T., Miller, D., Brown, L., \& Cross, P. (1985). A psychological analysis of cancer patients and their next-of-kin. Cancer, 55, 72-76. doi:10.1002/1097-0142(19850101)55:1<72:: AID-CNCR2820550112>3.0.CO;2-S

Charlson, M., Szatrowski, T. P., Peterson, J., \& Gold, J. (1994). Validation of a combined comorbidity index. Journal of Clinical Epidemiology, 47, 1245-1251. doi:10.1016/0895-4356(94)90129-5

Cohen, S. (2004). Social relationships and health. American Psychologist, 59, 676-684. doi:10.1037/0003-066X.59.8.676

Cohen, S., Doyle, W., \& Baum, A. (2006). Socioeconomic status is associated with stress hormones. Psychosomatic Medicine, 68, 414420. doi:10.1097/01.psy.0000221236.37158.b9

Cohen, S., Kamarck, T., \& Mermelstein, R. (1983). A global measure of perceived stress. Journal of Health and Social Behavior, 24, 385-396. doi: $10.2307 / 2136404$

Cohen, S., \& Wills, T. A. (1985). Stress, social support, and the buffering hypothesis. Psychological Bulletin, 98, 310-357. doi:10.1037/00332909.98.2.310

Compas, B., Worsham, N., Epping-Jordan, J., Grant, K., Mireault, G., Howell, D., \& Malcarne, V. (1994). When mom or dad has cancer: Markers of psychological distress in cancer patients, spouses, and children. Health Psychology, 13, 507. doi:10.1037/0278-6133.13.6.507

Connell, J., Whitworth, J., Davies, D., Lever, A., Richards, A., \& Fraser, R. (1987). Effects of acth and cortisol administration on blood pressure, electrolyte metabolism, atrial natriuretic peptide and renal function in normal man. Journal of Hypertension, 5, 425-433. doi:10.1097/ 00004872-198708000-00007

Cox, B., Richardson, A., Graham, P., Gislefoss, R. E., Jellum, E., \& Rollag, H. (2010). Breast cancer, cytomegalovirus and Epstein-Barr virus: A nested case-control study. British Journal Cancer, 102, 1665-1669. doi:10.1038/sj.bjc.6605675

Demark-Wahnefried, W., Morey, M. C., Clipp, E. C., Pieper, C. F., Snyder, D. C., Sloane, R., \& Cohen, H. J. (2003). Leading the way in exercise and diet (project lead): Intervening to improve function among older breast and prostate cancer survivors. Controlled Clinical Trials, 24, 206-223. doi:10.1016/S0197-2456(02)00266-0
Dobnig, H., Pilz, S., Scharnagl, H., Renner, W., Seelhorst, U., Wellnitz, B., ... Maerz, W. (2008). Independent association of low serum 25 hydroxyvitamin $\mathrm{d}$ and 1,25-dihydroxyvitamin $\mathrm{d}$ levels with all-cause and cardiovascular mortality. Archives of Internal Medicine, 168, 13401349. doi:10.1001/archinte. 168.12 .1340

Edwards, B., \& Clarke, V. (2004). The psychological impact of a cancer diagnosis on families: The influence of family functioning and patients' illness characteristics on depression and anxiety. Psycho Oncology, 13, 562-576. doi:10.1002/pon.773

Epplein, M., Zheng, Y., Zheng, W., Chen, Z., Gu, K., Penson, D., \& Shu, X. (2010). Quality of life after breast cancer diagnosis and survival. Journal of Clinical Oncology, 29, 406-412.

Gallo, L., Bogart, L., Vranceanu, A., \& Matthews, K. (2005). Socioeconomic status, resources, psychological experiences, and emotional responses: A test of the reserve capacity model. Journal of Personality and Social Psychology, 88, 386-399. doi:10.1037/0022-3514.88.2.386

Glaser, R., \& Kiecolt-Glaser, J. K. (1994). Stress-associated immune modulation and its implications for reactivation of latent herpesviruses. In R. Glaser \& J. Jones (Eds.), Human herpesvirus infections (pp. 245-270). New York, NY: Dekker.

Glaser, R., Litsky, M. L., Padgett, D. A., Baiocchic, R. A., Yang, E. V., Chen, M., ... Williams, M. V. (2006). Ebv-encoded dutpase induces immune dysregulation: Implications for the pathophysiology of ebvassociated disease. Virology, 346, 205-218. doi:10.1016/j virol.2005.10.034

Gorman, B., \& Sivaganesan, A. (2007). The role of social support and integration for understanding socioeconomic disparities in self-rated health and hypertension. Social Science \& Medicine, 65, 958-975. doi:10.1016/j.socscimed.2007.04.017

Gottlieb, B., \& Bergen, A. (2009). Social support concepts and measures. Journal of Psychosomatic Research, 69, 511-520.

Grassi, G., Cattaneo, B., Seravalle, G., Lanfranchi, A., \& Mancia, G. (1998). Baroreflex control of sympathetic nerve activity in essential and secondary hypertension. Hypertension, 31, 68-72.

Hegel, M., Moore, C., Collins, E., Kearing, S., Gillock, K., Riggs, R., ... Ahles, T. (2006). Distress, psychiatric syndromes, and impairment of function in women with newly diagnosed breast cancer. Cancer, 107. 2924-2931. doi:10.1002/cncr.22335

Henle, W., \& Henle, G. (1981). Epstein-Barr virus-specific serology in immunologically comprised individuals. Cancer Research, 41, 42224225.

Kannel, W. (1999). Historic perspectives on the relative contributions of diastolic and systolic blood pressure elevation to cardiovascular risk profile. American Heart Journal, 138, S205-S210. doi:10.1016/S00028703(99)70311-X

Kiecolt-Glaser, J., Dura, J., Speicher, C., Trask, O., \& Glaser, R. (1991). Spousal caregivers of dementia victims: Longitudinal changes in immunity and health. Psychosomatic Medicine, 53, 345-362.

Kiecolt-Glaser, J. K., Fisher, L. D., Ogrocki, P., Stout, J. C., Speicher, C. E., \& Glaser, R. (1987). Marital quality, marital disruption, and immune function. Psychosomatic Medicine, 49, 31-34.

Kiecolt-Glaser, J. K., Garner, W., Speicher, C., Penn, G. M., Holliday, J., \& Glaser, R. (1984). Psychosocial modifiers of immunocompetence in medical students. Psychosomatic Medicine, 46, 7-14.

Krause, N. (1997). Received support, anticipated support, social class, and mortality. Research on Aging, 19, 387-422. doi:10.1177/ 0164027597194001

Krause, N., \& Borawski-Clark, E. (1995). Social class differences in social support among older adults. The Gerontologist, 35, 498-508. doi: 10.1093/geront $/ 35.4 .498$

Kuo, C. P., Wu, C. L., Ho, H. T., Chen, C. G., Liu, S. I., \& Lu, Y. T (2008). Detection of cytomegalovirus reactivation in cancer patients receiving chemotherapy. Clinical Microbiology and Infection, 14, 221227. doi:10.1111/j.1469-0691.2007.01895.x 
Lin, N., \& Dean, A. (1984). Social support and depression. Social Psychiatry and Psychiatric Epidemiology, 19, 83-91.

Marmot, M., Fuhrer, R., Ettner, S., Marks, N., Bumpass, L., \& Ryff, C. (1998). Contribution of psychosocial factors to socioeconomic differences in health. Milbank Quarterly, 76, 403-448. doi:10.1111/14680009.00097

Matthews, K. A., \& Gallo, L. C. (2011). Psychological perspectives on pathways linking socioeconomic status and physical health. Аnnual Review of Psychology, 62, 501-530.

Morin, C. M. (1993). Insomnia: Psychological assessment and management. New York, NY: Guilford Press.

Morozink, J. A., Friedman, E. M., Coe, C. L., \& Ryff, C. D. (2010). Socioeconomic and psychosocial predictors of interleukin-6 in the midus national sample. Health Psychology, 29, 626-635. doi:10.1037/ a0021360

Nahmias, A., \& Roizman, B. (1973). Infection with herpes-simplex viruses 1 and 2. New England Journal of Medicine, 289, 781-789. doi:10.1056/ NEJM197310112891505

Neuling, S., \& Winefield, H. (1988). Social support and recovery after surgery for breast cancer: Frequency and correlates of supportive behaviours by family, friends and surgeon. Social Science \& Medicine, 27, 385-392. doi:10.1016/0277-9536(88)90273-0

Procidano, M. E., \& Heller, K. (1983). Measures of perceived social support from friends and from family: Three validation studies. American Journal of Community Psychology, 11, 1-24. doi:10.1007/ BF00898416

Radloff, L. S. (1977). The CES-D scale: A self-report depression scale for research in the general population. Applied Psychological Measurement, 1, 385-401. doi:10.1177/014662167700100306

Riley, D., \& Eckenrode, J. (1986). Social ties: Subgroup differences in costs and benefits. Journal of Personality and Social Psychology, 51, 770-778. doi:10.1037/0022-3514.51.4.770

Roberts, E. T., Haan, M. N., Dowd, J. B., \& Aiello, A. E. (2010). Cytomegalovirus antibody levels, inflammation, and mortality among elderly latinos over 9 years of follow-up. American Journal of Epidemiology, 172, 363-371. doi:10.1093/aje/kwq177

Roberts, R., \& Vernon, S. (1983). The center for epidemiologic studies depression scale: Its use in a community sample. American Journal of Psychiatry, 140, 41-46.

Savard, M., Savard, J., Simard, S., \& Ivers, H. (2005). Empirical validation of the insomnia severity index in cancer patients. Psycho-Oncology, 14, 429-441. doi:10.1002/pon.860

Simanek, A. M., Dowd, J. B., \& Aiello, A. E. (2009). Persistent pathogens linking socioeconomic position and cardiovascular disease in the US International Journal of Epidemiology, 38, 775-787. doi:10.1093/ije/ dyn 273

Steptoe, A., Shamaei-Tousi, A., Gylfe, A., Henderson, B., Bergstrom, S., \& Marmot, M. (2007). Socioeconomic status, pathogen burden and cardiovascular disease risk. Heart, 93, 1567-1570. doi:10.1136/ hrt.2006.113993

Stowe, R., Peek, M., Perez, N., Yetman, D., Cutchin, M., \& Goodwin, J. (2010). Herpesvirus reactivation and socioeconomic position: A community-based study. Journal of Epidemiology and Community Health, 64, 666-671. doi:10.1136/jech.2008.078808

Turner, R., \& Marino, F. (1994). Social support and social structure: A descriptive epidemiology. Journal of Health and Social Behavior, 35 193-212. doi: $10.2307 / 2137276$

Vitaliano, P. P., Scanlan, J. M., Zhang, J., Savage, M. V., Brummett, B., Barefoot, J., \& Siegler, I. C. (2001). Are the salutogenic effects of social supports modified by income? A test of an "added value hypothesis". Health Psychology, 20, 155-165. doi:10.1037/0278-6133.20.3.155

Winkleby, M., Jatulis, D., Frank, E., \& Fortmann, S. (1992). Socioeconomic status and health: How education, income, and occupation contribute to risk factors for cardiovascular disease. American Journal of Public Health, 82, 816-820. doi:10.2105/AJPH.82.6.816

Witek-Janusek, L., Gabram, S., \& Mathews, H. L. (2007). Psychologic stress, reduced nk cell activity, and cytokine dysregulation in women experiencing diagnostic breast biopsy. Psychoneuroendocrinology, 32, 22-35. doi:10.1016/j.psyneuen.2006.09.011

\section{E-Mail Notification of Your Latest Issue Online!}

Would you like to know when the next issue of your favorite APA journal will be available online? This service is now available to you. Sign up at http://notify.apa.org/ and you will be notified by e-mail when issues of interest to you become available! 